\title{
SUPERNOVA REMNANTS IN THE AKARI FIS ALL-SKY SURVEY
}

\author{
Il-Gyo JeOng ${ }^{1}$, Bon-Chul KoO ${ }^{1}$, And Ho-Gyu LeE ${ }^{2}$ \\ ${ }^{1}$ Department of Physics and Astrnomy, Seoul National University, Seoul 151-747, Korea \\ ${ }^{2}$ Department of Astronomy, Graduate School of Science, The University of Tokyo, 7-3-1 Hongo, Bunkyo-ku, \\ Tokyo 113-0033, Japan \\ E-mail: igjeong@astro.snu.ac.kr,koo@astro.snu.ac.kr \\ (Received July 08, 2012; Accepted July 23, 2012)
}

\begin{abstract}
We carry out a systematic study of Galactic supernova remnants (SNRs) using the AKARI Far Infrared Surveyor (FIS) survey data. The AKARI Infrared Astronomical Satellite observed the whole sky using the four FIS bands covering 50 to 180 microns with $\sim 1$ arcmin resolution. The allsky coverage with high-spatial resolution provides an unprecedented opportunity to study diffuse, extended far-infrared (FIR) sources such as SNRs. We have searched for FIR counterparts to all 274 known Galactic SNRs, and investigate their FIR properties of identified SNRs. We report preliminary results of the study.
\end{abstract}

Key words: ISM: supernova remnants; infrared: ISM

\section{INTRODUCTION}

The AKARI Far Infrared Surveyor (FIS) has performed the all-sky survey in four far-infrared (FIR) wavelength bands (N60, WIDE-S, WIDE-L, and N160). It covers the spectral range from 50 to 180 micron, and the spatial resolutions are $0^{\prime} .7$ for the N60 and WIDE-S bands, and about $1^{\prime}$ for the WIDE-L and N160 bands (Kawada et al. 2007). The scan area covers $99.1 \%$ of the sky.

In previous FIR studies of SNRs using the Infrared Astronomical Satellite (IRAS) all-sky survey data, Arendt et al. (1989) detected 30 out of 157 known SNRs. Saken et al. (1992) also found 35 out of 161 . Their study had a limitation for the analysis of small SNRs $\left(<30^{\prime}\right)$ because of low resolution. Additionally, the longest wavelength band of 100 micron wavelength was not enough to define the spectral energy distributions (SEDs) for the estimation of dust properties of SNRs.

The AKARI FIS data (Version 1.0) can give important information to understand the FIR properties of SNRs; FIR is useful for studying the cold dust formed during the process of supernova explosion (Sibthorpe et al., 2010; Barlow et al., 2010), FIR emission from the swept-up dust is the major coolant of an SNR and plays a role in the SNR evolution (Dwek et al., 1987), and FIR emission is enhanced when a SNR interacts with dense interstellar medium (ISM) (Drain, 1981; Lee et al., 2011).

In this proceeding paper we will show the preliminary results of our FIS survey of SNRs.

\section{PRELIMINARY RESULTS}

\subsection{Detection Rate of FIR SNRs}

We compare the 90 micron AKARI FIS images with the radio continuum images in order to identify FIR features associated with SNRs. The high-resolution of AKARI enables us to reveal small-scale FIR features that we have not seen before. According to our preliminary results, 63 out of 274 SNRs show bright FIR features.

\subsection{FIR Bright SNR}

Enhanced FIR emissions toward SNRs could be strong evidence for a shocked dense region (Drain, 1981; Lee 


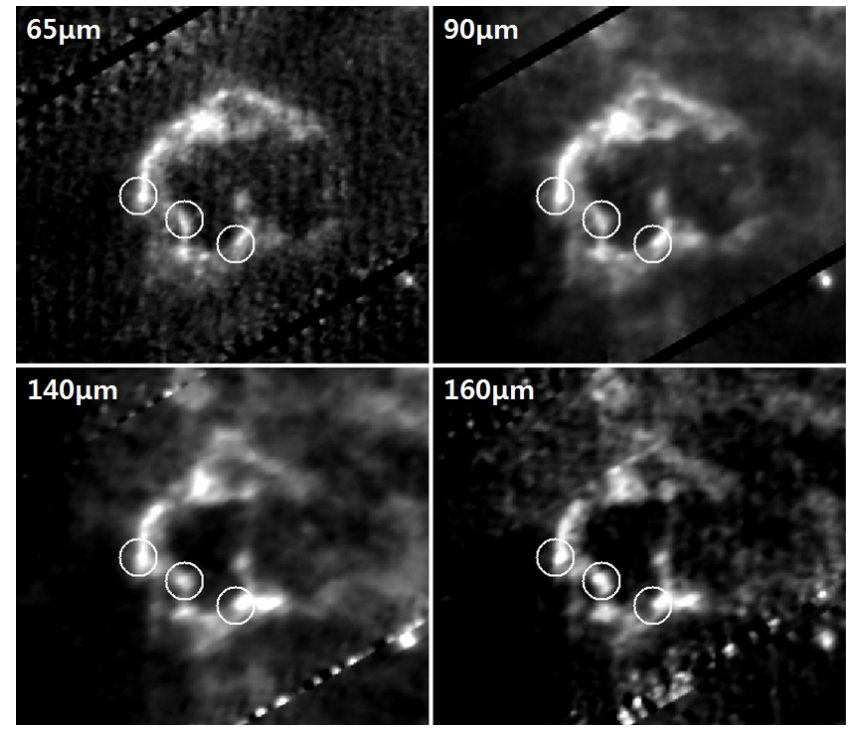

Fig. 1. AKARI FIS image of IC443 (G189.1+3.0). The areas marked by circles are where the molecular-line emission from shocked ambient cloud is prominent.

et al., 2011). Figure 1 shows the AKARI FIS images of IC443 in the four FIR bands. The size of IC 443 is about $45^{\prime}$ corresponding to about $20 \mathrm{pc}$ at a distance of $1.5 \mathrm{kpc}$ (Fesen 1984). According to the multiwavelength studies, IC443 shows multiple shell structures interacting with the ISM (Braun \& Strom, 1986; Mufson et al., 1986). In CO observation, it shows broad $\mathrm{CO}$ emission lines in the shocked regions (Snell et al., 2005; Lee et al., 2012). We mark the prominent shocked molecular regions in Figure 1. We can see that the bright FIR emission shows spatial agreement with the shocked molecular region. The enhanced FIR emission can have several origins: the dust emission from the inter-clump medium of shocked molecular clouds, the dust emission in evaporating flows of molecular clouds engulfed by hot gas, and the dust emission of nearby molecular clouds illuminated by radiative shocks (Lee et al., 2011). The AKARI FIS survey data will be very useful for studying SNRs interacting with molecular gas, providing information about both the gas and dust components associated with the shock interactions.

\section{ACKNOWLEDGEMENTS}

This research was supported by Basic Science Research Program through the National Research Foundation of Korea (NRF) funded by the Ministry of Education, Science and Technology (NRF-2011-0007223).

\section{REFERENCES}

Arendt, R. G., 1989, An Infrared Survey of Galactic Supernova Remnants, ApJS, 70, 181

Braun, R. \& Strom, R. G., 1986, Observations of PostShock Neutral Hydrogen in Four Evolved Supernova Remnants, A\&AS, 63, 345

Draine, B. T., 1981, Infrared Emission From Dust in Shocked Gas, ApJ, 245, 880

Dwek, E., Petre, R., Szymkowiak, A., \& Rice, W. L., 1987, IRAS Observations of Supernova Remnants - A Comparison between their Infrared and X-ray Cooling Rates, ApJ, 320, L27

Fesen, R. A,. 1984, The Nature of the Filaments Northeast of the Supernova Remnant IC 443, ApJ, 281, 658

Kawada, M., et al., 2007, The Far-Infrared Surveyor (FIS) for AKARI, PASJ, 59S, 389K

Lee, H. -G., Moon, D. -S., Koo, B. -C., Onaka, T., Jeong, W. -S., Shinn, J. -H., \& Sakon, I., 2011, Far-Infrared Luminous Supernova Remnant Kes 17, ApJ, 740, 31L

Lee, J. -J., Koo, B. -C., Snell, R. L., Yun, M. S., Heyer, M. H., \& Burton, M. G., 2012, Identification of Ambient Molecular Clouds Associated with Galactic Supernova Remnant IC 443, ApJ, $749,34 \mathrm{~L}$

Mufson, S. L., McCollough, M. L., Dickel, J. R., Petre, R., White, R., \& Chevalier, R., 1986, A Multiwavelength Investigation of the Supernova Remnant IC 443, AJ, 92, 1349

Saken, J. M., Fesen, R. A., \& Shull, J. M., 1992, An IRAS Survey of Galactic Supernova Remnants, ApJS, 81, 715

Snell, R. L., Hollenbach, D., Howe, J. E., Neufeld, D. A., Kaufman, M. J., Melnick, G. J., Bergin, E. A., \& Wang, Z., 2005, Detection of Water in the Shocked Gas Associated with IC 443: Constraints on Shock Models, ApJ, 620, 758S

Sibthorpe, et al., 2010, AKARI and BLAST Observations of the Cassiopeia A Supernova Remnant and Surrounding Interstellar Medium, ApJ, 719, $1553 \mathrm{~S}$ 